\title{
Intestinal motility distal of a deviating ileostomy after rectal resection with the construction of a primary anastomosis: results of the prospective COLO-MOVE study
}

\author{
T. A. Burghgraef ${ }^{1,2}$ (D) - F. J. Amelung ${ }^{3}$ • P. M. Verheijen ${ }^{1}$ - I. A. M. J. Broeders ${ }^{1}$ • E. C. J. Consten ${ }^{1,2}$
}

Accepted: 20 May 2020 / Published online: 5 June 2020

(C) The Author(s) 2020

\begin{abstract}
Purpose No consensus exists regarding the use of preoperative bowel preparation for patients undergoing a low anterior resection (LAR). Several comparative studies show similar outcomes when a single time enema (STE) is compared with mechanical bowel preparation (MBP). It is hypothesized that STE is comparable with MBP due to a decrease in intestinal motility distal of a newly constructed diverting ileostomy (DI).

Methods In this prospective single-centre cohort study, patients undergoing a LAR with primary anastomosis and DI construction were given a STE $2 \mathrm{~h}$ pre-operatively. Radio-opaque markers were inserted in the efferent loop of the DI during surgery, and plain abdominal X-rays were made during the first, third, fifth and seventh postoperative day to visualize intestinal motility.

Results Thirty-nine patients were included. Radio-opaque markers were situated in the ileum or right colon in 100\%, 100\% and $97.1 \%$ of the patients during respectively the first, third and fifth postoperative day. One patient had its most distal marker situated in the left colon during day five. In none of the patients, the markers were seen distal of the anastomosis.

Conclusion Intestinal motility distally of the DI is decreased in patients who undergo a LAR resection with the construction of an anastomosis and DI, while preoperatively receiving a STE.
\end{abstract}

Keywords Rectal neoplasms $\cdot$ Gastrointestinal motility $\cdot$ Bowel preparation

\section{Introduction}

In colorectal resections, bowel preparation is used to clean the colon of faeces, thereby preventing the passage of faeces across the anastomosis, aiming to reduce anastomotic leakage. Mechanical bowel preparation (MBP) can safely be omitted in colon resections, despite a recent suggestion of using the combination of oral antibiotics and MBP $[1,2]$. However, discussion

T. A. Burghgraef and F. J. Amelung contributed equally to this work.

T. A. Burghgraef

ta.burghgraef@meandermc.nl

1 Department of Surgery, Meander Medical Center, Maatweg 3, 3813, TZ Amersfoort, the Netherlands

2 Department of Surgery, University Medical Center Groningen, Hanzeplein 1, 9713, GZ Groningen, the Netherlands

3 Department of Surgery, University Medical Center, Utrecht, the Netherlands remains regarding the use of bowel preparation in rectal cancer surgery due to the high risk of anastomotic leakage [2].

MBP is not harmless: hypovolemia, electrolyte imbalances, renal failure and discomfort for the patient have been reported [3, 4]. In addition, MBP might not reduce gut microbial flora, but liquefy faeces, thereby increasing the risk of spillage and intraabdominal contamination [5]. Despite these adverse effects, omission of colon preparation does not seem feasible, since a randomized controlled trial showed MBP to be favourable over no bowel preparation in rectal cancer patients [3].

Single time enema (STE) has been suggested as an alternative for bowel preparation, as it is less burdensome and not associated with the above-mentioned complications associated with MBP [2]. Two studies show similar (infectious) outcomes when STE is compared with MBP in patients who underwent a low anterior resection (LAR) with the construction of a primary anastomosis and diverting ileostomy (DI) $[6,7]$.

This indicates that, even though faeces remain distal of the DI following preparation with an STE, it does not cause an increase in complication rate. Leading to the hypothesis that, intestinal motility distal of a newly constructed DI is decreased 
or even halted in the early postoperative phase. This prevents faeces to pass the anastomosis, resulting in comparable (infectious) complications, while only administering a STE.

Intestinal motility distal of a DI has already been shown to be decreased in patients that underwent a rectal resection with pre-operative MBP. However, it remained unclear whether the decrease in intestinal motility was due to MBP and the subsequent lack of faeces or a direct consequence of the DI [8]. This study aims to verify that intestinal motility is decreased after rectal resection and construction of a DI, when a STE is given as pre-operative bowel preparation.

\section{Materials and methods}

A prospective single-centre study was performed assessing intestinal motility distal of a DI in patients who underwent a LAR with the subsequent construction of a primary anastomosis and DI.

\section{Patients}

Study candidates were consecutively recruited and included if they (1) were older than 50 years, (2) had rectal carcinoma, (3) underwent an elective rectal resection with primary anastomosis, (4) received a DI, (5) received pre-operative STE and (6) gave informed consent. Exclusion criteria were (1) previous colonic resection, (2) known gastro-intestinal motility disorder, (3) allergy for gelatine or plastic or (4) a contra-indication for the use of rectal enema. Patients younger than 50 years were excluded to minimize the possible long-term consequences of additional radiation during the study. Patients with gastro-intestinal motility disorder were excluded since this would affect postoperative motility of the intestine.

\section{Procedures}

All study participants received a 'low' STE (Microlax, Johnson \& Johnson, Amersfoort, the Netherlands) $2 \mathrm{~h}$ preoperatively. A robot-assisted low anterior resection was performed. Following completion of a primary anastomosis, a DI was constructed. Twenty-four radiopaque markers were placed in the efferent loop of the DI.

Transit of the markers trough the colon was determined by plain abdominal X-rays taken at the first, third, fifth and seventh day postoperatively. Additional abdominal imaging, performed during follow-up, was included in the analysis. The location and number of markers were registered (terminal ileum/right colon, left colon, (neo)rectum or excreted). Intestinal motility was defined as a change in location of the most distal marker. Furthermore, patient characteristics, 30day morbidity and mortality and the occurrence of faeces excreted through the rectum as observed by nursing staff or the patient were determined.

\section{Statistical analysis}

Statistical analysis was done using R version 3.5.1. Continuous variables were expressed as mean and standard deviation (SD), or median and interquartile range (IQR), depending on the distribution. Categorical data were presented as absolute numbers and percentages.

\section{Results}

\section{Patient characteristics}

Between February 2016 and March 2019, 99 eligible patients were approached and 87 patients gave informed consent. However, 37 patients did not receive a DI or did not undergo a LAR. Furthermore, markers were inadequately placed in nine patients and two patients refused to undergo the postoperative X-rays, resulting in 39 included patients.

Patients had a median age of 65 , a median BMI of 24.4, $66.7 \%$ was male, $89.7 \%$ of the patients were scored as ASA III and $10.3 \%$ as ASA III. The tumour was located between 0 and $5 \mathrm{~cm}$ of the anal verge in $23.1 \%$, and between 5 and $10 \mathrm{~cm}$ in $69.2 \%$ of the patients. Neoadjuvant (chemo)radiation was received by $52.0 \%$ of the patients.

\section{Abdominal imaging}

In patients who underwent abdominal imaging during day one and day three, $100 \%$ of the markers were seen. In patients who underwent imaging during day five, one patient showed that the most distal marker migrated to the left colon (Table 1).

Rectal excretion of faeces postoperatively, with the DI in situ, was reported in $28.2 \%$ of the patients. In two patients, this occurred within 14 days postoperatively: At the fourth and the eleventh postoperative day. The patient reporting rectal excretion of faeces on the fourth postoperative day had an anastomotic leakage with faecal peritonitis, but without marker movement on the abdominal X-rays.

\section{Postoperative outcome}

No 30-day mortality was observed. Thirty-day major morbidity, defined as Clavien-Dindo classes III and IV, was seen in six patients $(15.4 \%)$. Five had an anastomotic leakage. This was treated conservatively with antibiotics in two patients, two patients required radiological drainage of a presacral abscess and one patient had to undergo a re-laparotomy with the construction of a new colostomy. A completely dehiscent anastomosis was seen during the re-laparotomy, with stapler 
Table 1 Location of most distal markers

\begin{tabular}{llll}
\hline Time & Imaging & Marker location \\
\hline Day 1 $(n, \%)$ & $37 / 39(94.9 \%)$ & Right colon & $37(100 \%)$ \\
& & Left colon & $0(0 \%)$ \\
Day 3 $(n, \%)$ & $36 / 39(92.3 \%)$ & Right colon & $36(100 \%)$ \\
& & Left colon & $0(0 \%)$ \\
Day 5 $(n, \%)$ & $34 / 39(87.2 \%)$ & Right colon & $33(97.1 \%)$ \\
& & Left colon & $1(2.9 \%)$ \\
Day 7 $(n, \%)$ & $15 / 39(38.5 \%)$ & Right colon & $14(93.3 \%)$ \\
& & Left colon & $1(7.1 \%)$ \\
3 months $(n, \%)$ & $21 / 39(53.8 \%)$ & Right colon & $8(38.1 \%)$ \\
& & Left colon & $4(19.0 \%)$ \\
& & (Neo) rectum & $1(4.8 \%)$ \\
& & Excreted & $8(38.1 \%)$ \\
\hline
\end{tabular}

remnants and faecal peritonitis. Marker movement in patients with an anastomotic leakage $(n=5)$ was absent.

\section{Discussion and conclusion}

This study aimed to provide information regarding the intestinal motility distal of a newly constructed ileostomy in patients undergoing a LAR with primary anastomosis, while pre-operatively receiving a STE. The results suggest that intestinal motility distal of the DI is absent or decreased in the majority of patients at least up until the fifth postoperative day.

Ali et al. found intestinal motility distal of a DI to be decreased in patients who underwent a LAR with the construction of a DI, while receiving MBP pre-operatively [8]. However, since these patients underwent MBP, it was unclear whether the halted intestinal motility was caused by the lack of faeces present in the colon or due to the construction of a deviating ileostomy. Huang et al. reported decreased motility in ten patients following LAR with primary anastomosis and DI construction while pre-operatively receiving a STE [9]; however, the sample size was low. Our study confirms the suggestion of Huang et al. and Ali et al. In addition, we show that patients preoperatively subjected to STE show decreased motility as well.

The mechanisms responsible for the decrease in intestinal motility remain unclear. Intestinal motility consists of segmental propagated contractions, which are arbitrarily divided into low-amplitude propagated contractions (LAPC) and high-amplitude propagated contractions (HAPC) [10]. The latter play a significant role in the oro-aboral shift of colonic content and require an intact enteric nervous system. In addition, it has been suggested that intraluminal continuity is necessary for intestinal motility $[11,12]$. Perhaps HAPCs are interrupted due to lack of intraluminal continuity.
Although intestinal motility distal of a DI is decreased, certain findings should be taken into account. First, this study is not a comparative study; therefore, our results are only applicable for patients who underwent rectal resection with primary anastomosis and DI, while preoperatively receiving a STE.

Secondly, the patient who had an anastomotic leakage with faecal peritonitis did not show intestinal motility on the plain abdominal X-rays, although faeces was excreted anally four days postoperative. The presence of faecal content in the abdominal cavity is in contrast with our hypothesis that intestinal motility distal of a DI is halted following DI construction. Since none of the markers migrated in this patient, perhaps the total dehiscent anastomosis facilitated intra-abdominal spillage of faecal content, especially in combination with gravitational force. More importantly, we used 'low' STE's which can only clean the rectum and the distal sigmoid. 'High' STEs might be necessary to prevent (passive) passage of faeces across the anastomosis by gravitational force.

Despite these limitations, the majority of patients did not show signs of intestinal motility distal of the DI on abdominal $\mathrm{X}$-ray. Subsequently, this study suggests a decrease or even a halt in intestinal motility distal of a DI in patients receiving a DI in the context of a LAR with primary anastomosis. Since bowel preparation is aimed at prevention of faecal passage across the anastomosis, these results suggest that a 'high' STE might be sufficient with decreased intestinal motility distal of a DI. However, a comparison between STE and MBP in patients receiving a LAR with primary anastomosis and a DI is necessary to confirm this.

Acknowledgements We thank the stoma nurses of the Meander Medical Center (L. Boerman \& N.E. van den Broek) for their contribution to the study.

Authors' contributions Study conception and design were performed by Amelung, Verheijen, Broeders and Consten. Burghgraef and Amelung performed the data acquisition. Analysis and interpretation, as well as preparing the original draft, were done by Burghgraef. Critical revision and editing of the draft and the final approval of the manuscript were done by all authors.

Data availability Anonymized patient data is available.

\section{Compliance with ethical standards}

Conflict of interest Broeders reports personal fees from Johnson \& Johnson and Intuitive Surgical. Consten and Verheijen report personal fees from Intuitive Surgical. Amelung and Burghgraef have no conflicts of interest or financial ties to disclose. No funding was received for this study.

Ethics approval The study was approved by the regional medical ethics committee (MEC-U, NL54504.100.15) and by the local institutional review board of the hospital. The study was performed in accordance with the declaration of Helsinki. 
Consent to participate Written informed consent was obtained from all patients who met inclusion criteria and were willing to participate.

Consent to publish Patients signed informed consent regarding publishing their data.

Open Access This article is licensed under a Creative Commons Attribution 4.0 International License, which permits use, sharing, adaptation, distribution and reproduction in any medium or format, as long as you give appropriate credit to the original author(s) and the source, provide a link to the Creative Commons licence, and indicate if changes were made. The images or other third party material in this article are included in the article's Creative Commons licence, unless indicated otherwise in a credit line to the material. If material is not included in the article's Creative Commons licence and your intended use is not permitted by statutory regulation or exceeds the permitted use, you will need to obtain permission directly from the copyright holder. To view a copy of this licence, visit http://creativecommons.org/licenses/by/4.0/.

\section{References}

1. Frontali A, Panis Y (2019) Bowel preparation in colorectal surgery: back to the future? Vol. 71, Updates in Surgery. Springer-Verlag Italia s.r.l., p 205-7

2. Guenaga K, Matos D, Wille-Jørgensen P (2011) Mechanical bowel preparation in elective colorectal surgery. J Med Sci. 19(1):31-34

3. Bretagnol F, Panis Y, Rullier E, Rouanet P, Berdah S, Dousset B, et al (2010) Rectal cancer surgery with or without bowel preparation: the french greccar III multicenter single-blinded randomized trial. In: Annals of Surgery. p 863-7

4. Hookey LC, Depew WT, Vanner S (2005) The safety profile of oral sodium phosphate for colonic cleansing before colonoscopy in adults. Gastrointest Endosc [cited 2019 May 15];56(6):895-902. Available from: https://www.sciencedirect.com/science/article/pii/ S0016510702703672?via\%3Dihub
5. Mahajna A, Krausz M, Rosin D, Shabtai M, Hershko D, Ayalon A, Zmora O (2005) Bowel preparation is associated with spillage of bowel contents in colorectal surgery. Dis Colon Rectum. 48(8): 1626-1631

6. Pittet O, Nocito A, Balke H, Duvoisin C, Clavien PA, Demartines N, Hahnloser D (2015) Rectal enema is an alternative to full mechanical bowel preparation for primary rectal cancer surgery. Color Dis. 17(11):1007-1010

7. Bertani E, Chiappa A, Biffi R, Bianchi PP, Radice D, Branchi V, et al (2011) Comparison of oral polyethylene glycol plus a large volume glycerine enema with a large volume glycerine enema alone in patients undergoing colorectal surgery for malignancy: a randomized clinical trial. Color Dis [cited 2019 May 23];13(10): 327-34. Available from: http://www.ncbi.nlm.nih.gov/pubmed/ 21689356

8. Ali JM, Rajaratnam SG, Upponi S, Hall NR, Fearnhead NS (2015) Colonic transit in the empty colon after defunctioning ileostomy: do we really know what happens? Tech Coloproctol. 19(3):165-172

9. Huang S, Theophilus M, Cui J, Bell SW, Wale R, Chin M, Farmer C, Warrier SK (2015) Colonic transit: what is the impact of a diverting loop ileostomy? ANZ J Surg. 87(10):795-799

10. Bassotti G, Iantorno G, Fiorella S, Bustos-Fernandez L, Bilder CR (1999) Colonic motility in man: features in normal subjects and in patients with chronic idiopathic constipation. Am J Gastroenterol [cited 2019 May 15]; 94(7):1760-70. Available from: http://www. ncbi.nlm.nih.gov/pubmed/10406232

11. Hallgren T, Öresland T, Cantor P, Fasth S, Hultén L (1995) Intestinal intraluminal continuity is a prerequisite for the distal bowel motility response to feeding. Scand J Gastroenterol [cited 2019 May 15]; 30(6):554-61. Available from: http://www.tandfonline. com/doi/full/10.3109/00365529509089789

12. Williams L, Armstrong M, Finan P, Sagar P, Burke D, First GO (2007) The effect of faecal diversion on human ileum. [cited 2019 May 15]; Available from: http://gut.bmjjournals.com/ifora/licence. dtl

Publisher's note Springer Nature remains neutral with regard to jurisdictional claims in published maps and institutional affiliations. 